\title{
GPU-Accelerated Montgomery Exponentiation
}

\author{
Sebastian Fleissner \\ Department of Computer Science and Engineering \\ The Chinese University of Hong Kong \\ seb@cse.cuhk.edu.hk
}

\begin{abstract}
The computing power and programmability of graphics processing units (GPUs) has been successfully exploited for calculations unrelated to graphics, such as data processing, numerical algorithms, and secret key cryptography. In this paper, a new variant of the Montgomery exponentiation algorithm that exploits the processing power and parallelism of GPUs is designed and implemented. Furthermore, performance tests are conducted and the suitability of the proposed algorithm for accelerating public key encryption is discussed.
\end{abstract}

Keywords: GPGPU, Montgomery Exponentiation, Encryption.

\section{Introduction}

Chips on consumer graphics cards have evolved into programmable graphics processing units (GPUs) capable of delivering real-time, photo-realistic effects in computer games and multimedia applications. According to an article by Manocha [1, the computational power of GPUs has a growth rate faster than Moore's law as it applies to other microprocessors. As a result, researchers attempt to exploit the processing power and programmability of GPUs for general purpose computing.

The purpose of this research is to investigate and realize the usage of GPUs for accelerating the Montgomery exponentiation algorithm, which is used by various public key encryption schemes, such as RSA and elliptic curves, for performing modular exponentiation of large integers. In particular, a new, GPU-accelerated Montgomery exponentiation algorithm is proposed and the performance of its implementation is evaluated. Furthermore, the suitability of the proposed GPU algorithm for accelerating public key encryption is discussed.

\subsection{Paper Organization}

The rest of the paper is organized as follows. Section 2 provides background information. Sections 3 and 4 specify and evaluate the proposed GPU-accelerated Montgomery exponentiation algorithm. Section 5 briefly discusses public key encryption schemes that are likely to benefit from the proposed algorithm. 


\section{Background}

\subsection{General Purpose GPU Computations}

General purpose GPU computing (GPGPU) 22 refers to the concept of exploiting the processing power of GPUs for performing general purpose calculations. Examples for GPGPU computing are data processing [3], evolutionary algorithms 4, and secret key cryptography [5].

A typical GPGPU application consists of two parts. One part, which is denoted as the main program, runs on the computer's main processor and is responsible for initializing the GPU, providing the GPU with input, and retrieving the results produced by the GPU. The other part, which is denoted as the fragment program, runs on the GPU and performs the desired general purpose calculation. The main program provides the operands for multiple calculations that are performed on the GPU in parallel. Since GPUs have only limited support for integer values, all data sent to and retrieved from GPUs is encoded using 32-bit floating point values.

The main program and fragment program exchange data via textures. Hence, the first step performed by the main program is to create several $2 \mathrm{D}$ textures inside the graphics memory, which serve as input and output buffers. After their creation, the textures serving as input buffers are filled with the operands for the general purpose calculation. After the textures are prepared, the main program invokes the fragment program on the GPU by drawing a rectangle with the same dimension as the textures. The GPU runs multiple instances of the fragment program in parallel, and each instance retrieves and processes the operands for a different calculation from the input textures. The results calculated by the fragment program instances are output as one or more 32 bit floating point values. These values are passed to the GPU processing pipeline and automatically stored in one or more designated output textures. The program on the main processor can then access the output textures to obtain the results.

\subsection{The Montgomery Method}

The Montgomery method [6] and its various improvements [78|9] are algorithms for efficient computation of modular multiplications $x=a \times b \bmod n$ and modular exponentiations $x=a^{b} \bmod n$, with $a, b, n$ being k-bit large integers and $n$ being odd.

As described in 10, the Montgomery algorithm consists of following steps:

\section{Perform pre-computation}

Choose a large integer $r$ as a power of 2, with $r>n$. Then $n^{\prime}$ is calculated, so that $r \times r^{-1}-n \times n^{\prime}=1$. Both $r^{-1}$ and $n^{\prime}$ can be calculated using the extended Euclidian algorithm.

\section{Obtain Montgomery representation of $\mathbf{a}$ and $\mathbf{b}$}

This step uses the $r$ generated during the pre-computation step to transform the operands $a$ and $b$ into Montgomery representation. Their Montgomery representations are obtained by calculating $\bar{a}:=a \times r \bmod n$ and $\bar{b}:=b \times$ $r \bmod n$. 


\section{Calculate Montgomery Product $\overline{\mathbf{x}}=\overline{\mathbf{a}} \times \overline{\mathbf{b}}$}

This step calculates the Montgomery product $\operatorname{MonPro}(\bar{a}, \bar{b})=\bar{x}:=\bar{a} \times \bar{b} \times r^{-1}$ $\bmod n$. The Montgomery product is calculated as follows:
(a) $t:=\bar{a} \times \bar{b}$
(b) $m:=t \times n^{\prime} \bmod r$
(c) $x:=(t+m \times n) / r$
(d) if $x \geq n$ then set $x=x-n$

Montgomery Exponentiation. Because of the overhead caused by the precomputation and representation transformation steps, the Montgomery method is used for modular exponentiation rather than a single modular multiplication. A common form of the Montgomery exponentiation algorithm, which is described in [10], uses the so-called binary square-and-multiply method to calculate $x=a^{b}$ mod $n$, where $a, b, n$ are $k$-bit large integers and $n$ is odd. With $|b|$ being the bit length of operand $b$, the algorithm consists of the following steps:

1. Use $n$ to pre-compute $n^{\prime}$ and $r$.

2. Calculate $\bar{a}:=a \times r \bmod n$.

3. Calculate $\bar{x}:=1 \times r \bmod n$.

4. For $\mathrm{i}:=|b|-1$ down to 0 do

(a) Calculate $\bar{x}:=\operatorname{MonPro}(\bar{x}, \bar{x})$

(b) If the $i$-th bit of $b$ is set, then calculate $\bar{x}:=\operatorname{MonPro}(\bar{a}, \bar{x})$

5. Calculate $x=\operatorname{MonPro}(\bar{x}, 1)$.

\section{Proposed Algorithm}

\subsection{Overview}

This section proposes a new, GPU-accelerated exponentiation algorithm based on the Montgomery method introduced in section 2.2 This algorithm, denoted as GPU-MonExp, exploits the parallelism of GPUs by calculating multiple modular exponentiations simultaneously. The exponentiation operands have a fixed bit size, which depends on the output capabilities of the GPU hardware.

Like the Montgomery exponentiation algorithm introduced in section 2.2, the GPU-MonExp algorithm depends on the Montgomery product. As a result, this section first describes a GPU-accelerated variant of the Montgomery product denoted as GPU-MonPro, which forms the basis of the GPU-MonExp algorithm.

As GPUs have limited support for integer values, the proposed GPU algorithms split large integers into $k$ 24-bit chunks, and store each chunk in a 32-bit floating point value. The first chunk contains the least significant bits and the last chunk contains the most significant bits of the large integer. Hence, the representation of a large integer $\mathrm{x}$ is:

$$
x=x[0], x[1], \ldots, x[k]
$$




\subsection{GPU Montgomery Product(GPU-MonPro)}

The GPU-MonPro algorithm utilizes the GPU to calculate $c$ Montgomery products in parallel:

$$
\overline{x_{i}}:=\overline{a_{i}} \times \overline{b_{i}} \times r^{-1} \bmod n_{i}, 1 \leq i \leq c
$$

The GPU-MonPro algorithm uses large integers with a pre-defined, fixed bit size. Because of the fixed bit size, the maximum size of the $n_{i}$ operands is known, and by considering that $r>n$ and $r=2^{z}$ for some $z$, the value of the operand $r$ can be pre-defined as well in order to simplify calculations. As graphics processing units do not provide any efficient instructions for performing bitwise operations, the GPU-MonPro algorithm uses an operand $r$ that is a power of 256 . Thus, multiplication and division operations by $r$ can be implemented via byte shifting, which can be performed efficiently by GPUs. Since the large integers used by the algorithms in this chapter consist of $k$ 24-bit chunks, the value of $r$ is pre-defined as $r=256^{3 k}$.

As $r$ is pre-defined, the input values for the GPU-MonPro algorithm are $\overline{a_{i}}, \overline{b_{i}}$, $n_{i}, n_{i}^{\prime}$. These input values are supplied by the GPU-MonExp algorithm described in section 3.3 .

The GPU-MonPro algorithm consists of two steps: Texture preparation and calculation of the Montgomery product.

Step 1: Texture preparation [Main Processor]. The GPU-MonPro algorithm uses multiple two-dimensional input and output textures in RGBA color format. An RGBA texel (texture element) consists of four 32-bit floating point values and can thus be used to encode four 24-bit chunks (96 bit) of a large integer. The algorithm uses four types of input textures corresponding to the four types of operands: tex- $\bar{a}$, tex- $\bar{b}$, tex- $n$, and tex- $n$. Assuming input textures with a dimension of $w \times h$ to calculate $c=w \times h$ Montgomery products, the GPU-MonPro algorithm uses the following approach to store the operands $\bar{a}_{i}$, $\bar{b}_{i}, n_{i}, n_{i}^{\prime}$ in the input textures:

1. For each $0 \leq x<w, 0 \leq y<h$ do:

2. $i:=y \times w+x$

3. Store $\bar{a}_{i}$ in the tex- $\bar{a}$ texture(s)
(a) tex- $\left.\bar{a}_{[0]}(\mathrm{x}, \mathrm{y})=\bar{a}_{i}[0,1,2,3]\right]^{1}$
(b) tex-a $\bar{a}_{[1]}(\mathrm{x}, \mathrm{y})=\bar{a}_{i}[4,5,6,7]$
(c) $\ldots$
(d) $\operatorname{tex}-\bar{a}_{[k / 4]}(\mathrm{x}, \mathrm{y})=\bar{a}_{i}[k-4, k-3, k-2, k-1]$

4. Store $\bar{b}_{i}$ in the tex- $\bar{b}$ texture $(\mathrm{s})$
(a) tex-b $\bar{b}_{[0]}(\mathrm{x}, \mathrm{y})=\bar{b}_{i}[0,1,2,3]$
(b) $\ldots$
(c) tex- $\bar{b}_{[k / 4]}(\mathrm{x}, \mathrm{y})=\bar{b}_{i}[k-4, k-3, k-2, k-1]$

5 . Store $n_{i}$ in the tex- $n$ texture(s)
(a) tex- $n_{[0]}(\mathrm{x}, \mathrm{y})=n_{i}[0,1,2,3]$
(b) ...
(c) $\operatorname{tex}-n_{[k / 4]}(\mathrm{x}, \mathrm{y})=n_{i}[k-4, k-3, k-2, k-1]$

$\overline{1}$ The term $\bar{a}_{i}[0,1,2,3]$ is an abbreviation for the four values $\bar{a}_{i}[0], \bar{a}_{i}[1], \bar{a}_{i}[2], \bar{a}_{i}[3]$. 
6. Store $n_{i}^{\prime}$ in the tex- $n^{\prime}$ texture $(\mathrm{s})$

(a) tex- $n_{[0]}^{\prime}(\mathrm{x}, \mathrm{y})=n_{i}^{\prime}[0,1,2,3]$

(b) $\ldots$

(c) $\operatorname{tex}-n_{[k / 4]}^{\prime}(\mathrm{x}, \mathrm{y})=n_{i}^{\prime}[k-4, k-3, k-2, k-1]$

Considering a large integer as $\mathrm{k}$ 24-bit chunks, the total number of textures required for storing the operands $\bar{a}_{i}, \bar{b}_{i}, n_{i}, n_{i}^{\prime}$ is $\frac{k}{4} 4=k$. (There are four operands and each texel can store four 24-bit chunks). The number of required output textures is $\frac{k}{4}$.

After the input and output textures are prepared, drawing commands are issued in order to invoke the fragment program instances on the GPU.

Step 2: Calculation of Montgomery product [GPU]. Each instance of the fragment program on the GPU calculates one modular product. Since the GPU hardware runs multiple instances of the fragment program in parallel, several modular products are calculated at the same time. Apart from the input textures containing the $\overline{a_{i}}, \overline{b_{i}}, n_{i}$, and $n_{i}^{\prime}$ values, each fragment program instance receives a $(\mathrm{X}, \mathrm{Y})$ coordinate pair that indicates which operands $\bar{a}, \bar{b}, n$, and $n^{\prime}$ should be retrieved from the input textures to calculate the Montgomery product.

The algorithm performed by the fragment program instances, which is essentially a standard Montgomery multiplication, is as follows:

1. Use the $\mathrm{X}$ and $\mathrm{Y}$ coordinates to obtain the four operands $\overline{a_{j}}, \overline{b_{j}}, n_{j}$, and $n_{j}^{\prime}$ for some specific $j$, with $1 \leq j \leq c$.

2. Calculate $t:=\overline{a_{j}} \times \overline{b_{j}}$. Because the maximum bit size of the operands is pre-defined, multiplication can be implemented efficiently on GPUs using vector and matrix operations, which are capable of calculating multiple partial products in parallel.

3. Calculate $m:=t \times n_{j}^{\prime} \bmod r$. Since $r$ is a multiple of 256 , the reduction by modulo $r$ is achieved by byte shifting.

4. Calculate $\bar{x}:=\left(t+m \times n_{j}\right) / r$. By using the vector and matrix operations of the GPU, addition can be implemented efficiently, since partial sums can be calculated in parallel. The division by $r$ is achieved by byte shifting.

5 . Output $\bar{x}$, which is automatically diverted and stored in the output textures.

\subsection{GPU Montgomery Exponentiation (GPU-MonExp)}

The GPU-MonExp algorithm calculates $c$ modular exponentiations in parallel:

$$
x_{i}=a_{i}^{b} \bmod n_{i}, 1 \leq i \leq c
$$

Each of the $c$ exponentiations uses the same $b$, but different values for each $a_{i}$ and $n_{i}$. With $|b|$ denoting the fixed bit size of operand $b$, the GPU-MonExp algorithm executes the following steps: 
1. Execute the following loop on the main processor:

For $\mathrm{i}:=1$ to $\mathrm{c}$ do

(a) Use $n_{i}$ to pre-compute $n_{i}^{\prime}$.

(b) Calculate $\bar{x}_{i}:=1 \times r \bmod n_{i}$.

(c) Calculate $\overline{a_{i}}:=a_{i} \times r \bmod n_{i}$.

2. Execute the following loop on the main processor:

For $l:=|b|-1$ down to 0 do

(a) Invoke GPU-MonPro to calculate

$\bar{x}_{i}:=\bar{x}_{i} \times \bar{x}_{i} \times r^{-1} \bmod n_{i}$ on the GPU in parallel. $(1 \leq i \leq c)$

(b) If the $l$-th bit of $b$ is set, then invoke GPU-MonPro to calculate $\bar{x}_{i}:=$ $\bar{a}_{i} \times \bar{x}_{i} \times r^{-1} \bmod n_{i}$ on the GPU in parallel. $(1 \leq i \leq c)$

3. Invoke GPU-MonPro to calculate the final results

$x_{i}=\bar{x}_{i} \times 1 \times r^{-1} \bmod n_{i}$ on the GPU in parallel. $(1 \leq i \leq c)$

Step 1 Details. The pre-computation loop, which is executed on the main processor, calculates a corresponding $n_{i}^{\prime}$ for each $n_{i}$ using the extended Euclidian algorithm. If all modular products to be calculated use the same $n$, then only one $n^{\prime}$ is computed, since $n^{\prime}$ only depends on $n$ and not on the multiplicand and multiplier. Apart from the $n_{i}^{\prime}$ values, the loop determines the initial values for all $\bar{x}_{i}$ and the Montgomery representations of all $a_{i}$.

Step 2 Details. The main loop of the GPU-MonExp algorithm is run on the main processor and uses the square-and-multiply approach to calculate the modular exponentiations. The main loop first invokes GPU-MonPro with the $\bar{x}_{i}$ values as texture parameters in order to calculate the squares $\bar{x}_{i} \times \bar{x}_{i} \times r \bmod$ $n_{i}$ on the GPU in parallel. Depending on whether the current bit of operand $b$ is set, the algorithm invokes GPU-MonPro again with $\bar{x}_{i}$ and $\bar{a}_{i}$ as texture parameters to calculate the Montgomery products $\bar{x}_{i} \times \bar{a}_{i} \times r \bmod n_{i}$ on the GPU in parallel. After the main loop completes, the final results $x_{i}=a_{i}^{b} \bmod$ $n_{i}$ are transferred back to the main processor.

\section{Algorithm Evaluation}

\subsection{Performance Test Overview}

This section introduces and analyzes the results of performance tests, which were conducted in order to evaluate the potential of the proposed GPU-MonExp algorithm. In order to obtain representative test data, three different hardware configurations were used to run the performance tests. The details of these three test systems, which are denoted as system A, B, and C, are shown in table 1 .

The GPU-MonExp performance test works as follows. As a first step, the implementation of the GPU-MonExp algorithm is run with random 192-bit operands for 1 to 100000 exponentiations. The execution time $T_{G P U}$ is measured and recorded. Then an implementation of the square-and-multiply Montgomery exponentiation algorithm described in section 2.2 is run with the same input 
Table 1. Test Systems

\begin{tabular}{|c|l|l|}
\hline System & Processor/Memory/Graphics Bus & GPU \\
\hline $\mathrm{A}$ & Intel Pentium 4, 2.66 GHz & NVIDIA GeForce 6500 \\
& 1 GB RAM, PCI-Express & 256 MB RAM \\
\hline B & Intel Celeron, 2.40 GHz & NVIDIA GeForce FX 5900 Ultra \\
& 256 MB RAM, AGP & 256 MB RAM \\
\hline C & Intel Pentium 4, 3.20GHz & NVIDIA GeForce 7800 GTX \\
& 1 GB RAM, PCI-Express & 256 MB RAM \\
\hline
\end{tabular}

on the computer's main processor, and its execution time, denoted as $T_{S Q M}$, is recorded as well. Using the execution times of both implementations, the following speedup factor of the GPU-MonExp algorithm is determined:

$$
s=\frac{T_{S Q M}}{T_{G P U}}
$$

If the implementation of the GPU-MonExp algorithm runs faster than the square-and-multiply Montgomery exponentiation, then its execution time is shorter and $s>1$.

The implementations of the GPU-MonExp and the underlying GPU-MonPro algorithm are based on OpenGL, C++, and GLSL (OpenGL Shading Language). The second step of GPU-MonPro described in section 3.2 is implemented as a GLSL fragment program. The parts of the GPU-MonExp algorithm running on the main processor are implemented in $\mathrm{C}++$.

\subsection{Test Results}

Overall, the test results indicate that the GPU-MonExp algorithm is significantly faster than the square-and-multiply Montgomery exponentiation, if multiple results are calculated simultaneously. When a large amount of modular exponentiations is calculated simultaneously, the GPU-MonExp implementation is 136 - 168 times faster.

Table 2. GPU-MonExp Speedup Factors

\begin{tabular}{|l|l|l|l|}
\hline Exponentiations & System A & System B & System C \\
\hline 1 & 0.2264 & 0.0783 & 0.1060 \\
3 & 1.2462 & 0.4843 & 0.8271 \\
10 & 4.0340 & 1.2878 & 2.9767 \\
50 & 19.5056 & 6.4544 & 13.2813 \\
100 & 33.2646 & 13.0150 & 25.6985 \\
10000 & 138.0390 & 110.9095 & 138.8840 \\
100000 & 168.9705 & 136.4484 & 167.1229 \\
\hline
\end{tabular}

As shown in table2. GPU-MonExp already achieves a performance gain when 3 to 10 exponentiations are calculated simultaneously. When calculating 100000 
exponentiations simultaneously, the speedup factors are 168.9705 for system A, 136.4484 for system B, and 167.1229 for system C.

\section{Conclusions}

This paper introduces the concept of using graphics processing units for accelerating Montgomery exponentiation. In particular, a new GPU-accelerated Montgomery exponentiation algorithm, denoted as GPU-MonExp, is proposed, and performance tests show that its implementation runs 136 - 168 times faster than the standard Montgomery exponentiation algorithm.

Public key encryption algorithms that are based on elliptic curves defined over prime fields (prime curves) are likely to benefit from the proposed GPU-MonExp algorithm, which can serve as the basis for GPU-accelerated versions of the point doubling, point addition, and double-and-add algorithms. Signcryption schemes based on elliptic curves, such as 1112, are a possible concrete application for the GPU-MonExp algorithm.

\section{References}

1. Manocha, D.: General-purpose computations using graphics processors. Computer 38(8) (August 2005) 85-88

2. Pharr, M., Fernando, R.: GPU Gems 2 : Programming Techniques for HighPerformance Graphics and General-Purpose Computation. Addison-Wesley (2005)

3. Govindaraju, N.K., Raghuvanshi, N., Manocha, D.: Fast and approximate stream mining of quantiles and frequencies using graphics processors. In: SIGMOD '05, New York, NY, USA, ACM Press (2005) 611-622

4. M. L. Wong, T.T.W., Foka, K.L.: Parallel evolutionary algorithms on graphics processing unit. In: IEEE Congress on Evolutionary Computation 2005. (2005) $2286-2293$

5. Cook, D., Ioannidis, J., Keromytis, A., Luck, J.: Cryptographics: Secret key cryptography using graphics cards (2005)

6. Montgomery, P.L.: Modular multiplication without trial division. Mathematics of Computation 44(170) (April 1985) 519-521

7. Gueron, S.: Enhanced montgomery multiplication. In: CHES '02, London, UK, Springer-Verlag (2003) 46-56

8. Walter, C.D.: Montgomery's multiplication technique: How to make it smaller and faster. Lecture Notes in Computer Science 1717 (1999) 80-93

9. WU, C.L., LOU, D.C., CHANG, T.J.: An efficient montgomery exponentiation algorithm for cryptographic applications. INFORMATICA 16(3) (2005) 449-468

10. Koc, C.K.: High-speed RSA implementation. Technical report, RSA Laboratories (1994)

11. Zheng, Y., Imai, H.: Efficient signcryption schemes on elliptic curves. In: Proc. of IFIP SEC'98. (1998)

12. Han, Y., Yang, X.: Ecgsc: Elliptic curve based generalized signcryption scheme. Cryptology ePrint Archive, Report 2006/126 (2006) 